\title{
Induced sepsis by cecal ligation and puncture in a rat model: hepatic and renal histological features
}

\author{
Adrian Florin $\mathrm{GAL}^{1}$, Sidonia BOGDAN ${ }^{1}$, Flavia RUXANDA ${ }^{1 *}$, Vasile RUS ${ }^{1}$ \\ ${ }^{1}$ University of Agricultural Sciences and Veterinary Medicine Cluj-Napoca, Faculty of Veterinary Medicine, \\ Calea Mănăștur 3-5, Cluj-Napoca, 400372, Romania \\ *corresponding author: flavia.ruxanda@usamvcluj.ro
}

Bulletin UASVM Veterinary Medicine 76(2)/2019

Print ISSN 1843-5270; Electronic ISSN 1843-5378

doi:10.15835/buasvmcn-vm:2019.0015

\begin{abstract}
:
Animal models have been developed in an attempt to test potential therapeutic agents. The study aims to determine the hepatic and renal histological features induced by sepsis following cecal ligation and puncture (CLP) in a rat model. Regarding the material, we used 20 adult-male Wistar rats (control group 1, n=10, and group 2, n=10, that underwent CLP protocol). The experimental protocol was approved by the National Sanitary Veterinary and Food Authority, Cluj (Romania), project number 116/11.05.2018. The endpoint of the experiment was pre-set to 10 hours post-surgery. A complete necropsy survey was performed. The main renal lesions detected histologically were: glomerular congestion and edema, hyalinization of glomerular mesangium, presence of hyaline in the urinary space with associated compression atrophy of vascular glomerular tuft, granules of hyaline in the lumen of cortical tubules, and reduction of the urinary space. The hepatic lesion identified histologically was represented by isolated miliary necrotic foci.
\end{abstract}

Keywords: histology, sepsis, model, puncture, rat.

\section{Introduction}

Sepsis is a severe condition defined as a "lifethreatening organ dysfunction that is caused by a dysregulated host response to infection". The immune response that is initiated in sepsis by an invading pathogen, fails to return to homeostasis and instead continues to develop, ultimately reaching a state of sustained excessive inflammation and immune suppression (Van Der Poll et al., 2017). Traditionally, the immune response to sepsis was thought to be composed of two separate phases: an initial hyperinflammatory phase lasting several days, followed by a more prolonged immunosuppressive phase. However, recent evidence suggests that early in the pathogenesis of sepsis, both proinflammatory and anti-inflammatory responses develop simultaneously (Hotchkiss et al., 2013), and based on the direction of imbalance, the pro- cess can result in inflammation and tissue damage, immunosuppression and infection, or recovery (Ettinger et al., 2016).

Animal models have been developed in an attempt to create reproducible systems, in order to study the pathogenesis of sepsis and to test potential therapeutic agents (Buras et al., 2005), since staging human sepsis models presents multiple difficulties (Fink and Heard, 1990; Deitch, 1998). In light of this evidence, the current study aims to determine the main hepatic and renal histological features that can be detected in induced sepsis by cecal ligation and puncture (CLP) in a rat model.

\section{Materials and methods}

In the study, we used 20 adult-male Wistar rats that were bought from the Faculty of Human 
Medicine and Pharmacy Iuliu Haţieganu ClujNapoca. During this period, the rats were kept in boxes in groups of ten, at $20-26^{\circ} \mathrm{C}$, with room humidity at around 40-70\%, in a $12 \mathrm{~h}$ light/dark cycle and fed with standard rat food and water ad libitum. The experimental protocol was approved by the National Sanitary Veterinary and Food Authority, Cluj (Romania), project number 116, on May $11^{\text {th }}, 2018$. The experimental study was conducted at the Faculty of Veterinary Medicine Cluj-Napoca (Romania). Ten rats represented the control group (i.e., group no. 1) and did not go under any procedure. The individuals from the second group (no. $=10$ ) underwent CLP protocol for sepsis induction (Wichterman et al., 1980; Parker and Watkins, 2001). Basically, the CLP protocol involves the perforation of the cecum with a 21G needle (following anesthesia and visualization of the cecum by standard abdominal surgery). Before cecal perforation, a ligature was applied distal to the ileocecal valve at $1 \mathrm{~cm}$ from the tip of the cecum. The needle penetrated the caecum on both sides performing 2 holes in the organ. The cecum was gently squeezed until its contents began to exude through the perforations and then it was repositioned in the abdominal cavity. Following the CLP procedure, the second group was maintained in normothermia (35.9$\left.37.5^{\circ} \mathrm{C}\right)$. The endpoint of the experiment was preset to 10 hours post-surgery, during this time the rats were clinically monitored. At the end of the experiment, the rats were euthanized using a high dose of Isoflurane followed by cervical dislocation. The sepsis was confirmed microbiologically.

Eventually, the necropsy exam was performed and the tissue samples (liver and kidney) were collected, underwent fixation in $10 \%$ buffered formalin and processed by classical paraffin technique and stained later by the H\&E method. The examination of the histological slides achieved was performed using an Olympus BX41 light microscope, and the images were captured with an Olympus E-330 photo camera, attached to the microscope.

\section{Results and discussion}

In contrast to the previous experimental studies which have only found minor histological changes (Brooks et al., 2007; (Buras et al., 2005; Deitch, 1998; Lerolle et al., 2010), in our experiment we observed renal injury that affected large portions of the kidneys. In group 2 , the rats showed glomerular congestion and edema, as well as a significant reduction of the urinary space associated with hyalinization of glomerular mesangium (Figure 1) and discrete intraglomerular infiltrate composed of young neutrophils. In addition, the tubular epithelium of the cortical tubules showed cloudy to vacuolar degeneration (Figure 2). Hyaline was also observed in the lumen of cortical tubules (Figure 3 ), and corticomedullary hyperemia was present. In some individuals $(n=3)$, the deposits of hyaline from the urinary space have led to compression atrophy of vascular glomerular tuft. In contrast to Lerolle et al. (2010) and Takasu et al. (2013), which found that most nephrons appeared normal, we observed that these changes affected the majority of corpuscles in the cortex.

Regarding the liver state in group 2 individuals, the lesions were represented by necrotic miliary foci (Figure 4). Each miliary focus of necrosis appeared to be coagulative necrosis of a small group of hepatocytes. Differently, from the findings published by Muftuoglu et al. (2006), the inflammatory reactions identified in liver samples from rats in the current study were only negligible.

The host barrier disruption model such as the CLP model is widely used and even considered the "golden standard" for sepsis research (Wichterman et al., 1980; Parker and Watkins, 2001). CLP in rats mimics the fundamental clinical features of human sepsis including the following development of multi-organ dysfunction. Therefore, it appears to be the best currently available animal model used in sepsis research. In the CLP model, the infective selection is uninterrupted due to the presence of a complete range of enteric pathogens in the septic focus. Hence, CLP resembles the clinical condition of bowel perforation, with the consequential infection caused by a bacterial mix originating in the intestine (Brooks et al., 2007). Several characteristics have been shown to exist in both rat CLP models and humans with sepsis, including progressive bacteremia, fever, increased metabolism and changes in blood flow (Wichterman et al., 1980).

\section{Conclusion}

The histological findings described in a range of sepsis-induced protocols are scarce. The main renal lesions detected histologically in CLP rat 


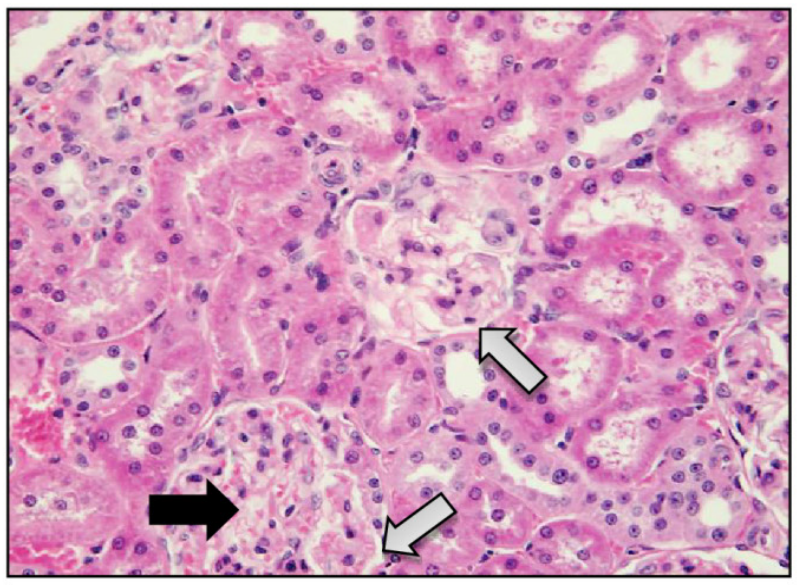

Figure 1. Glomerular congestion and edema

(black arrow); hyalinization of glomerular

mesangium associated with a reduction of the urinary space (grey arrows); Kidney, H\&E stain, ob. $40 \mathrm{x}$.

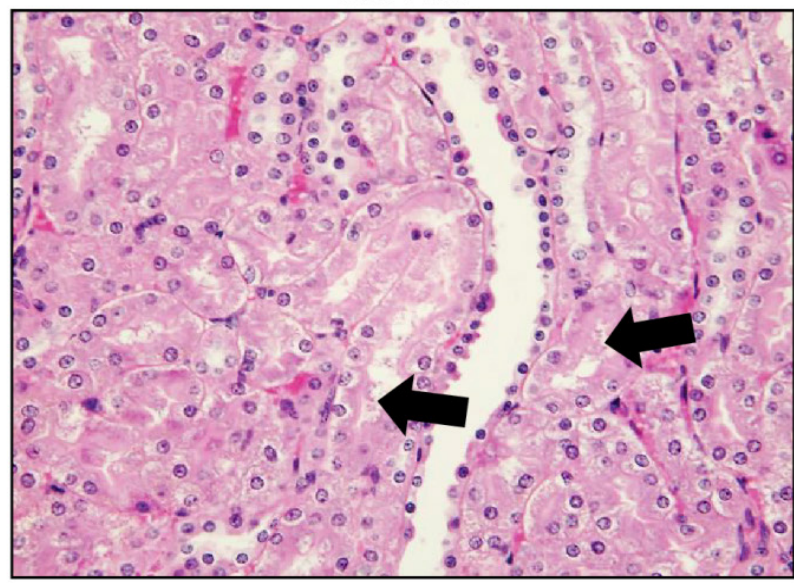

Figure 3. Presence of discrete proteinaceous material (hyaline) in the lumen of cortical tubules (black arrows); Kidney, H\&E stain, ob. 40x.

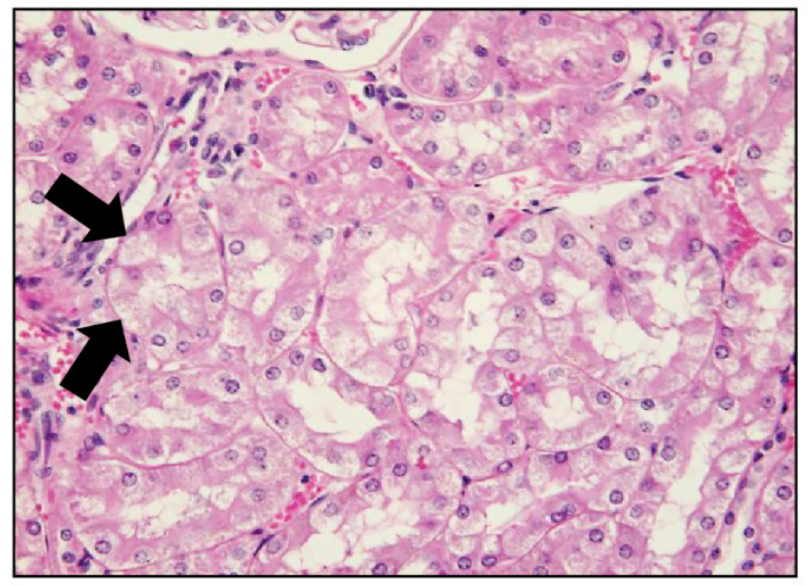

Figure 2. Cloudy to vacuolar nephrosis (black arrows) of the urinary epithelium of the nephron's tubules; Kidney, H\&E stain, ob. 40x.

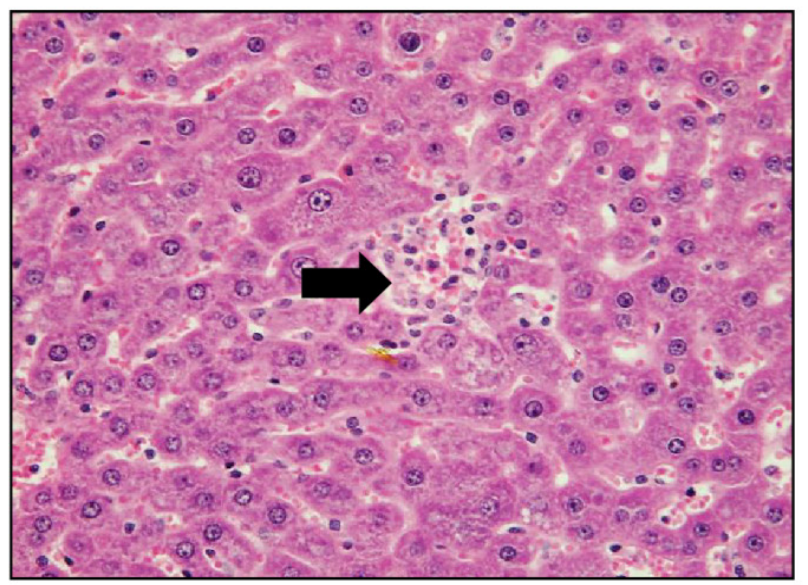

Figure 4. A miliary focus of hepatic necrosis with a negligible inflammatory reaction (black arrow); Liver, H\&E stain, ob. 40x. model of sepsis were: glomerular congestion and edema, hyalinization of glomerular mesangium, presence of hyaline in the urinary space with associated compression atrophy of vascular glomerular tuft, discrete granules of hyaline in the lumen of cortical tubules, and reduction of the urinary space. The hepatic lesion identified histologically was represented by isolated miliary necrotic foci.

Acknowledgments. This research did not receive any specific grant from funding agencies in the public, commercial, or not-for-profit sectors.

\section{References}

1. Brooks HF, Osabutey CK, Moss RF, Andrews PLR, Davies DC (2007). Caecal ligation and puncture in the rat mimics the pathophysiological changes in human sepsis and causes multi-organ dysfunction. Metab Brain Dis, 22: 353-373.

2. Buras JA, Holzmann B, Sitkovsky M (2005). Animal Models of sepsis: setting the stage. Nat Rev Drug Discov, 4: 854-865.

3. Deitch EA (1998). Animal models of sepsis and shock: a review and lessons learned. Shock (Augusta, Ga.), 9: 1-11.

4. Ettinger SJ, Feldman EC, Cote E (2016). Textbook of veterinary internal medicine: diseases of the dog and the cat. pp. 1492-1504.

5. Fink MP, Heard SO (1990). Laboratory models of sepsis and septic shock. J Surg Res, 49: 186-196. 
6. Hotchkiss RS, Monneret G, Payen D (2013). Sepsisinduced immunosuppression: from cellular dysfunctions to immunotherapy. Nat Rev Immunol, 13: 862-874.

7. Lerolle N, Nochy D, Guérot E, Bruneval P, Fagon J-YY, Diehl J-LL, Hill G (2010). Histopathology of septic shock induced acute kidney injury: Apoptosis and leukocytic infiltration. Intens Care Med, 36: 471-478.

8. Muftuoglu MAT, Aktekin A, Ozdemir NC, Saglam A (2006). Liver injury in sepsis and abdominal compartment syndrome in rats. Surg Today, 36: 519-524.

9. Parker SJ, Watkins PE (2001). Experimental models of Gram-negative sepsis. Brit J Surg, 88(1): 22-30.
10. Takasu O, Gaut JP, Watanabe E, To K, Fagley RE, Sato B, Jarman S, Efimov IR, Janks DL, Srivastava A, Bhayani SB, Drewry A, Swanson PE, Hotchkiss RS (2013). Mechanisms of cardiac and renal dysfunction in patients dying of sepsis. Am J Resp Crit Care, 187: 509-517.

11. Van Der Poll T, Van De Veerdonk FL, Scicluna BP, Netea MG (2017). The immunopathology of sepsis and potential therapeutic targets. Nat Rev Immunol, 17: 407-420.

12. Wichterman KA, Baue AE, Chaudry IH (1980). Sepsis and septic shock-a review of laboratory models and a proposal. J Surg Res, 29: 189-201. 